\title{
Efficient Privacy-Preserving Large-scale CP Tensor Decompositions
}

\author{
Changqing Luo ${ }^{1}$, Sergio Salinas ${ }^{2}$, and ${\mathrm{Pan} \mathrm{Li}^{3}}^{3}$ \\ ${ }^{1}$ Department of Computer Science, Virginia Commonwealth University, Richmond, VA 23284 \\ ${ }^{2}$ Department of Electrical Engineering and Computer Science, Wichita State University, Wichita, KS 67260 \\ ${ }^{3}$ Department of Electrical Engineering and Computer Science, Case Western Reserve University, Cleveland, OH 44106 \\ Email: cluo@vcu.edu, salinas@cs.wichita.edu, and lipan@case.edu.
}

\begin{abstract}
Tensor decompositions are very powerful tools for analyzing multi-dimensional multi-modal data. Particularly, CP tensor decomposition is one of the most fundamental tensor decomposition models. However, it is usually computationally expensive to conduct $\mathrm{CP}$ tensor decompositions on a largescale tensor by common algorithms like alternative least squares (ALS). To address this issue, one widely recognized solution is to adopt cloud computing. However, this raises privacy concerns due to the private information carried by a tensor. Previous algorithms for privacy-preserving outsourcing of tensor decompositions and other related computations require heavy communication cost. In this paper, we first develop an efficient tensor transformation scheme to protect the private information carried by elements' values of a tensor. Then we design a privacy-preserving outsourcing algorithm for ALS based CP tensor decompositions. We implement our proposed algorithm on a laptop and Amazon EC2 cloud and offer experiment results to show the significant computing time-savings.
\end{abstract}

Index Terms-Tensor decomposition, data privacy, big data analysis

\section{INTRODUCTION}

Tensor, a multi-dimensional array, has been widely used to characterize multi-dimensional multi-modal or multi-aspect data [1]. For instance, we can use a fourth-order tensor to represent the time-location-video data in smart cities [2]. We can use a third-order tensor to represent the patient-diagnosisprocedure dataset collected from healthcare and medical applications [3]. We can construct a third-order tensor to model the user-keywords-time data from online social networks [4]. Through

It is more convenient for people to find the underlying correlations among different aspects of their collected data through constructing and analyzing such tensor data. Tensor decompositions are very powerful tools for conducting tensor data analysis. For example, police officers apply tensor decompositions to analyze video footage for searching crime suspects [5]. Doctors use tensor decompositions to analyze health data from smart healthcare systems for identifying patients who have similar diagnosis and have undergone the similar procedures [3]. Marketing specialists employ tensor decompositions to analyze large-scale tensor data collected from online social networks to find better business opportunities [6]. We can see that people are able to obtain valuable information from various datasets by applying tensor decompositions.
Researchers have so far developed several tensor decomposition models used for diverse data analysis purposes. We notice that CANDECOMP/PARAFAC (CP) tensor decomposition [7] is one of the most widely used tensor decomposition models. Thus, we consider this model in this paper. The basic idea of CP tensor decompositions is to decompose a tensor into rank-1 tensors, which are called "factor matrices". To conduct $\mathrm{CP}$ tensor decompositions, researchers have proposed several algorithms [8]. One of the most popular algorithms is the Alternating Least Squares (ALS) algorithm [9], which is usually too computationally expensive to be used by users with limited computation resources [10]. As a consequence, this results in an obstacle in well analyzing massive data collected from the emerging various systems and applications.

To overcome this issue, one widely recognized solution is to employ cloud computing that is able to provide normal users or organizations with abundant computing and storage resources. Specifically, normal users or small organizations (i,e., called renters) can rent the cloud resources. Then, they can outsource their computations to the cloud which performs the computations on behalf of them and returns the computing results. In so doing, the renters can complete their tasks in the cloud while saving tremendous infrastructure investments. Hence, adopting cloud computing is economic as well.

However, adopting cloud computing incurs privacy concerns since the data usually contain very sensitive information that cannot be revealed due to ethical, legal, or security reasons. For example, video collected in smart city applications can reveal users' face information. Criminals can use this information for committing crimes [5]. Potential diseases that patients may suffer from can be extracted from the data collected in smart healthcare systems. Third-parties like advertisement companies and insurance companies have considerable interest in such health data [11]. Each user's life patterns can be revealed by the personal messages (e.g., visited places, visiting time, activities) that they posted in online social networks. Criminals are able to explore such data to commit crimes [12]. Therefore, to enable CP tensor decompositions on largescale data, we need to design an efficient privacy-preserving outsourcing algorithm for analyzing large-scale tensor data.

Previous works have studied the privacy-preserving outsourcing problem and proposed several schemes for them. More precisely, researchers develop linear data transformation 
techniques, such as mapping functions and linear algebra operations, to protect the data privacy. For example, Wang et al. [13] explore the affine mapping technique to transform a linear programming problem before outsourcing it to the cloud. Salinas et al. develop privacy-preserving outsourcing algorithms for solving LSEs [14], [15] and quadratic programming (QP) [16], respectively. Luo et al. explore speciallyshaped matrices to design secure outsourcing algorithms for matrix factorizations [17], [18], and employ the matrix addition to design secure outsourcing algorithm for nonlinearly constrained nonlinear programming problems [19]. Zhou and Li [20] explore matrix addition to mask a matrix used for eigen/singular value decomposition. Liao et al. [21], [22] exploit a matrix transformation technique to design an efficient privacy-preserving outsourcing algorithm for convex separable programming problems. We can see that such works usually require high communication overhead, and the efficient privacy-preserving outsourcing of large-scale tensor decompositions is still an open and challenging problem.

In this paper, we first develop a scheme to protect the privacy of a tensor data. In particular, we preserve tensor values by performing tensor modal product along with a mode with a carefully-designed pseudorandom sparse matrix. Then, based on the developed schemes, we design a privacypreserving outsourcing algorithm for ALS based CP tensor decompositions. Specifically, first, the renter conceals an original tensor by applying the designed privacy-preserving tensor transformation scheme to each mode of the tensor, and uploads the transformed tensor to the cloud. Afterwards, the cloud employs the ALS algorithm to decompose the transformed tensor and return the corresponding factor matrices. At last, the renter recovers real factor matrices from the returned ones. Due to only performing computations over sparse matrices, our proposed algorithm can achieve low computational complexity at the renter side. It takes the computations with $\mathcal{O}\left(K_{1} K_{2} \cdots K_{N}\right)$ complexity when performing CP tensor decompositions on a $K_{1} \times K_{2} \times \cdots \times K_{N}$ tensor with $d-$ rank. We implement and evaluate our proposed algorithm on a laptop and EC2 cloud. We notice that our proposed algorithm has very significant computing time savings.

We organize the rest of this paper as follows. We formulate the considered problem in Section II. We describe the privacypreserving tensor transformation scheme in Section III. Section IV presents the proposed privacy-preserving outsourcing algorithm for ALS based CP tensor decompositions. Section $\mathrm{V}$ provides a thorough analysis of the performance. We show the experiment results in Section VI. We finally conclude this paper in Section VII.

\section{PROBlem Formulation}

\section{A. System Architecture}

In this paper, we consider that a user with general-purpose computers rents cloud services to conduct $\mathrm{CP}$ tensor decompositions on a large-scale $N^{t h}$-order tensor $\mathcal{X}$ to obtain $N$

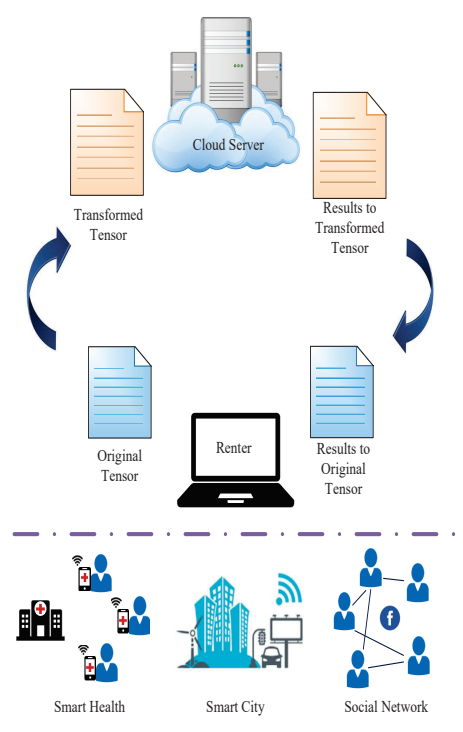

Fig. 1. A two-party computing architecture for privacy-preserving outsourcing of $\mathrm{CP}$ tensor decompositions.

loading factor matrices $A^{(1)}, A^{(2)}, \cdots, A^{(N)}$, i.e.,

$$
\begin{aligned}
\mathcal{X} & =\sum_{j=1}^{d} a_{j}^{(1)} \circ a_{j}^{(2)} \circ \cdots \circ a_{j}^{(N)} \\
& =\left[A^{(1)}, A^{(2)}, \cdots, A^{(N)}\right],
\end{aligned}
$$

where $\mathcal{X}$ is a $K_{1} \times K_{2} \times \cdots \times K_{N}$ tensor, $d$ is a tensor rank used to indicate the number of latent factors $A^{(n)} \mathrm{s}$, for $n \in[1, N]$, each of which is of size $K_{n} \times d, a_{j}^{n}$ is the $j$ th column of $A^{(n)}$, ० is the outer product operator, and [.] is the $\mathrm{CP}$ tensor decomposition operator. We consider that all the variables are integer, and all the computations are conducted within group $\mathbb{G}$.

To conduct $\mathrm{CP}$ tensor decompositions, we consider a twoparty asymmetric computing architecture as shown in Fig. 1. Specifically, to protect the privacy, the renter first transforms an original tensor and uploads the transformed one to the cloud. Then, the cloud performs CP tensor decompositions on its received tensor and returns factor matrices to the renter. At last, the renter recovers real factor matrices from the returned ones.

\section{B. Threat Model}

In this paper, we assume that elements' values in a tensor carry private information that needs to be protected. The cloud is "honest-but-curious", thereby wanting to learn users' private information from any data it can access, including the data received from the renter, intermediate computing results, and final factor matrices.

\section{Privacy-Preserving Tensor Transformation}

We propose to efficiently hide non-zero values in a tensor by employing tensor modal product, 
i.e., a tensor multiplied by a sparse pseudorandom matrix along a mode. Specifically, we conceal tensor $\mathcal{X}=\left(x_{i_{1}, i_{2}, \cdots, i_{N}}\right)_{1 \leq i_{1} \leq K_{1}, 1 \leq i_{2} \leq K_{2}, \cdots, 1 \leq i_{N} \leq K_{N}}$ and its $n$th factor matrix as follows:

$$
\hat{\mathcal{X}}=\mathcal{X} \times_{n}\left(I_{n}+S_{n} P_{n}\right)
$$

where $\times_{n}$ denotes the $n$-mode product, $I_{n}$ is a $K_{n} \times K_{n}$ identity matrix, $S_{n}$ represents a $K_{n} \times K_{n}$ diagonal pseudorandom matrix, and $P_{n}$ is a $K_{n} \times K_{n}$ sparse matrix.

Matrix $S_{n}$ is defined as follows:

$$
s_{i, j}=\left\{\begin{array}{cc}
t_{i}, & \text { for } 1 \leq i=j \leq K_{n} \\
0, & \text { otherwise }
\end{array}\right.
$$

Each diagonal element $t_{i} \in \mathbb{G}$ is generated by a pseudorandom function $F_{s}:\{0,1\}^{\omega} \times\{0,1\}^{\omega} \rightarrow\{0,1\}^{\omega}$, i.e.,

$$
t_{i}=F_{s}\left(r_{i}, g\right)
$$

where $r_{i}$ is a random string and $g$ is a constant.

Matrix $P_{n}$ is defined as follows:

$$
p_{i, j}=\left\{\begin{array}{cc}
q_{i, j}, & \text { if }(i, j) \in \mathcal{K}_{i_{n}} \\
0, & \text { otherwise }
\end{array}\right.
$$

for $i, j \in\left[1, K_{n}\right]$. We can set each $q_{i, j} \leftarrow\{0,1\}^{k}$ as an arbitrary integer within group $\mathbb{G}$. The set of index pairs $\mathcal{K}_{i_{n}}$ is determined by

$\mathcal{K}_{i_{n}}=\left\{\left(i_{n}, i_{j}\right) \mid q_{i_{n}, i_{j}} x_{i_{1} \cdots i_{j} \cdots i_{N}} \neq 0 \wedge i_{n} \neq i_{j}, i_{j} \in\left[1, K_{j}\right]\right\}$,

where $i_{n} \in\left[1, K_{n}\right]$. To limit the computational complexity of finding $\hat{\mathcal{X}}$, we set the maximum size of $\mathcal{K}_{i_{n}}$ to a small constant, i.e., $\left|\mathcal{K}_{i_{n}}\right| \ll K_{n}$ (for $i \in\left[1, K_{n}\right]$ ).

As a result, each non-zero element of tensor $\hat{\mathcal{X}}$, denoted by $\hat{x}_{i_{1}, i_{2}, \cdots, i_{N}}$, can be given by

$$
\begin{aligned}
\hat{x}_{i_{1}, \cdots, i_{n}, \cdots, i_{N}} & =x_{i_{1}, \cdots, i_{n}, \cdots, i_{N}} \\
& +t_{i_{n}} \sum_{\left(i_{n}, i_{j}\right) \in \mathcal{K}_{i_{n}}} q_{i_{n}, i_{j}} x_{i_{1}, \cdots, i_{j}, \cdots, i_{N}} .
\end{aligned}
$$

We denote these computations as

$$
\hat{x}_{i_{1}, i_{2}, \cdots, i_{N}}=\operatorname{Mask}^{F_{s}}\left(r_{i_{n}}, q_{i_{n}, i_{j}}, x_{i_{1}, i_{2}, \cdots, i_{N}}\right),
$$

for $\left(i_{n}, i_{j}\right) \in \mathcal{K}_{i_{n}}$.

We can transform a tensor by performing such computations, and protect the privacy of the original tensor and the corresponding $n$th factor matrix. This privacy-preserving tensor tranformation scheme will be employed to our privacypreserving outsourcing algorithm design.

\section{Privacy-Preserving OUtsourcing of CP Tensor DECOMPOSITIONS}

In this section, we introduce an ALS algorithm for CP tensor decompositions, and present the developed privacy-preserving outsourcing algorithm for CP tensor decompositions.

\section{A. Alternating Least Squares (ALS) Algorithm for CP Tensor Decompositions}

Before describing in detail our proposed privacy-preserving outsourcing algorithm, we first introduce an ALS algorithm commonly used for conducting $\mathrm{CP}$ tensor decompositions. The main idea of $\mathrm{CP}$ tensor decompositions is to find factor matrices that can be used to reconstruct a tensor best approximating the original one. Therefore, to obtain factor matrices of a specific tensor $\mathcal{X}$ with $d$ components, we need to solve the following optimization problem:

$$
\begin{array}{ll}
\min _{\mathcal{X}^{\prime}} \quad & f\left(\mathcal{X}^{\prime}\right)=\left\|\mathcal{X}-\mathcal{X}^{\prime}\right\|_{F}, \\
& \text { with } \mathcal{X}^{\prime}=\sum_{j=1}^{d} a_{j}^{(1)} \circ a_{j}^{(2)} \circ \cdots \circ a_{j}^{(N)} \\
& =\left[A^{(1)}, A^{(2)}, \cdots, A^{(N)}\right],
\end{array}
$$

where function $f(\cdot)$ is used to measure the distance between the original tensor $\mathcal{X}$ and the reconstructed one $\mathcal{X}^{\prime}$, and $\|\cdot\|_{F}$ is the Frobenius norm of a tensor.

Since $f\left(\mathcal{X}^{\prime}\right)$ is non-convex, researchers usually employ an ALS algorithm, an iterative optimization method, to solve (8). Its basic idea is to first reduce an original optimization problem into several smaller sub-problems and then solve these subproblems sequentially at an iteration. Specifically, the original optimization problem is first converted into an easily solvable least squares problem by fixing all but one factor matrix. Then, the corresponding factor matrix is found by solving the converted least squares problem. We follow the above procedure to sequentially update all factor matrices at each iteration and continue this updating process until a certain stopping condition is satisfied.

To be more specific, to solve (8) by the ALS algorithm, we first convert the original problem into $N$ least squares problems that are written in the matrix form as follows:

$$
\begin{gathered}
\min _{A^{(1)}}\left\|\mathcal{X}_{(1)}-A^{(1)}\left(A^{(N)} \odot A^{(N-1)} \odot \cdots \odot A^{(2)}\right)^{T}\right\|_{F}, \\
\vdots \\
\min _{A^{(n)}} \| \mathcal{X}_{(n)}-A^{(n)}\left(A^{(N)} \odot \cdots \odot A^{(n+1)} \odot\right. \\
\left.A^{(n-1)} \odot \cdots \odot A^{(1)}\right)^{T} \|_{F}, \\
\vdots \\
\min _{A^{(N)}}\left\|\mathcal{X}_{(N)}-A^{(N)}\left(A^{(N-1)} \odot A^{(N-2)} \odot \cdots \odot A^{(1)}\right)^{T}\right\|_{F},
\end{gathered}
$$

where $X_{(n)}$, for $n \in[1, N]$, are the unfolding matrix along the $n$-th mode, and " $\odot$ " is the Khatri-Rao product [8]. A sub-problem $\min _{A^{(n)}}\|\cdot\|_{F}$, for $n \in[1, N]$, is first generated by fixing $A^{(1)}, \cdots, A^{(n-1)}, A^{(n+1)}, \cdots, A^{(N)}$, and then solved to obtain a new $A^{(n)}$.

Through sequentially solving all sub-problems at an itera- 
tion, we can obtain the factor matrices as follows:

$$
\begin{gathered}
A^{(1)}=\mathcal{X}_{(1)}\left[\left(A^{(N)} \odot A^{(N-1)} \odot \cdots \odot A^{(2)}\right)^{T}\right]^{\dagger}, \\
\vdots \\
A^{(n)}=\mathcal{X}_{(n)}\left[\left(A^{(N)} \odot \cdots \odot A^{(n+1)} \odot\right.\right. \\
\left.\left.A^{(n-1)} \odot \cdots \odot A^{(1)}\right)^{T}\right]^{\dagger}, \\
\vdots \\
A^{(N)}=\mathcal{X}_{(N)}\left[\left(A^{(N-1)} \odot A^{(N-2)} \odot \cdots \odot A^{(1)}\right)^{T}\right]^{\dagger},
\end{gathered}
$$

where $[\cdot]^{\dagger}$ is the pseudoinverse of a matrix. In practice, it is difficult to find the pseudoinverse of a rectangular matrix. Fortunately, we can exploit the special form of the pseudoinverse of the Khatri-Rao product to rewrite (9). Based on the Khatri-Rao product, we have $\left(A^{(N)} \odot \cdots \odot A^{(n+1)} \odot A^{(n-1)} \odot\right.$ $\left.\left.\cdots \odot A^{(1)}\right)^{T}\right]^{\dagger}=\left(A^{(N)} \odot \cdots \odot A^{(n+1)} \odot A^{(n-1)} \odot \cdots \odot\right.$ $\left.A^{(1)}\right)\left(A^{(N) T} A^{(N)} * \cdots * A^{(n+1) T} A^{(n+1)} * A^{(n-1) T} A^{(n-1)} *\right.$ $\left.\cdots * A^{(1) T} A^{(1)}\right)^{\dagger}$, for $1 \leq n \leq N$, where “*” is the Hadamard product. For simple presentation, we have

$$
\begin{aligned}
V_{n} & =A^{(N) T} A^{(N)} * \cdots * A^{(n+1) T} A^{(n+1)} * \\
& A^{(n-1) T} A^{(n-1)} * \cdots * A^{(1) T} A^{(1)} \\
W_{n} & =A^{(N)} \odot \cdots \odot A^{(n+1)} \odot A^{(n-1)} \odot \cdots \odot A^{(1)},
\end{aligned}
$$

for $1 \leq n \leq N$. As a result, (9) can be rewritten as follows:

$$
\begin{gathered}
A^{(1)}=\mathcal{X}_{(1)} W_{1} V_{1}^{\dagger}, \\
\vdots \\
A^{(n)}=\mathcal{X}_{(n)} W_{n} V_{n}^{\dagger}, \\
\vdots \\
A^{(N)}=\mathcal{X}_{(N)} W_{N} V_{N}^{\dagger} .
\end{gathered}
$$

Thus, we only need to compute the inverse of a $d$-by-d matrix rather than the pseudoinverse of a $\left(K_{1} \cdots K_{n-1} K_{n+1} \cdots K_{N}\right)$-by-d matrix.

The ALS algorithm is to sequentially update $A^{(1)}, A^{(2)}, \cdots, A^{(N)}$ by using (10) at each iteration. This updating process will terminate until a certain stopping condition is met, i.e., the number of iterations exceeds a predefined maximum number $\mathbb{K}$. Therefore, we finally find the factor matrices, i.e., $A^{(1)}, A^{(2)}, \cdots, A^{(N)}$. We summarize the ALS algorithm for conducting CP tensor decompositions on tensor $\mathcal{X}$ in Algorithm 1.

\section{B. A Privacy-Preserving Outsourcing Algorithm for CP Ten- sor Decompositions}

Our proposed privacy-preserving outsourcing algorithm includes transforming an original tensor, conducting $\mathrm{CP}$ tensor decompositions over the transformed tensor, and recovering real factor matrices. We present them in detail as follows.

1) To transform an original tensor: To simultaneously protect the privacy of a tensor $\mathcal{X}$ and its factor matrices, the renter transforms $\mathcal{X}$ as follows:

$$
\overline{\mathcal{X}}=\mathcal{X} \times{ }_{1} U_{1} \cdots \times_{n} U_{n} \cdots \times_{N} U_{N}
$$

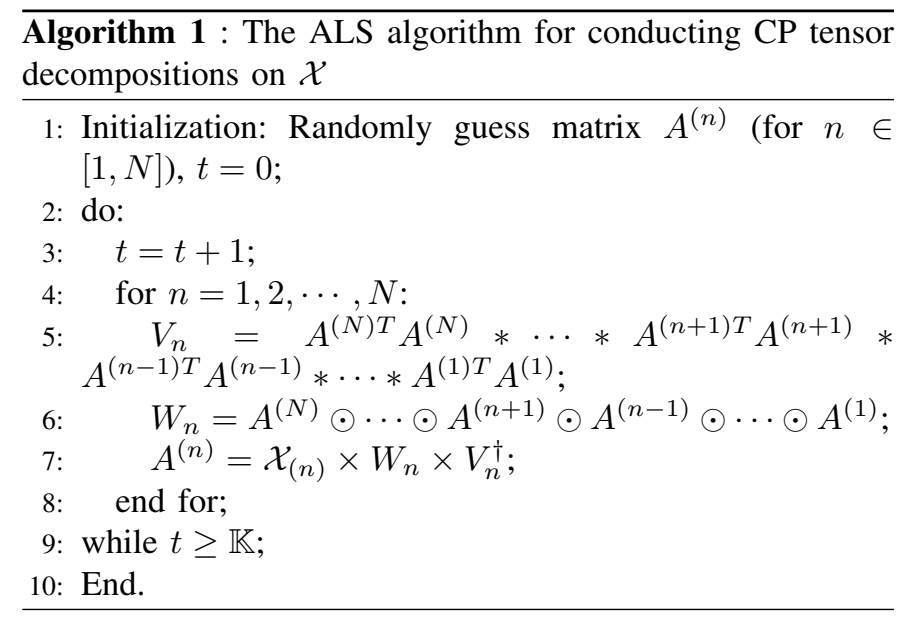

where $U_{n}=I_{n}+S_{n} P_{n}$, for $n \in[1, N]$.

Since $U_{n} \mathrm{~s}, \forall n \in[1, N]$, are sparse, performing tensor modal product between $\mathcal{X}$ and each $U_{n}$ only requires very low computational complexity. Hence, the renter is able to perform the computations of transforming tensor $\mathcal{X}$ by itself.

2) To conduct CP Tensor Decompositions: The cloud first receives the transformed tensor $\overline{\mathcal{X}}$ sent from the renter. Then it applies the ALS algorithm to conduct CP tensor decompositions on $\overline{\mathcal{X}}$ for obtaining the factor matrices, i.e.,

$$
\overline{\mathcal{X}}=\left[\bar{A}^{(1)}, \bar{A}^{(2)}, \cdots, \bar{A}^{(N)}\right],
$$

where $\bar{A}^{(n)}=U_{n} \times A^{(n)}$ (for $n \in[1, N]$ ). Specifically, the cloud carries out Algorithm 1 to find factor matrices $\bar{A}^{(n)}$, for $1 \leq n \leq N$. At last, the cloud returns these transformed factor matrices to the renter.

3) To recover real factor matrices: To find real factor matrices from the returned results, i.e., $\bar{A}^{(1)}, \bar{A}^{(2)}, \cdots, \bar{A}^{(N)}$, the renter performs computations as follows:

$$
A^{(n)}=U_{n}^{-1} \bar{A}^{(n)} \text {, for } n \in[1, N] .
$$

where $U_{n}^{-1}=\left(I_{n}+S_{n} P_{n}\right)^{-1}$

Since $\left(I_{n}+S_{n} P_{n}\right)$, for $1 \leq n \leq N$, are very sparse, the renter can complete the computations required by performing matrix inverse within a feasible period. Thus, the renter calls a matrix inverse algorithm to find the inverse of $\left(I_{n}+S_{n} P_{n}\right)$, for $1 \leq n \leq N$. Moreover, due to the sparsity of $\left(I_{n}+S_{n} P_{n}\right)^{-1}$, for $1 \leq n \leq N$, the renter also performs the matrix recovery to find real factor matrices by itself, instead of borrowing the help from the cloud.

\section{Performance Analysis}

In this section, we present the analysis of the computational complexity, communication complexity, and privacy, respectively.

\section{A. Computational Complexity}

We present a thorough analysis of the computational complexity as follows. In the process of finding $\overline{\mathcal{X}}$, the computations are conducted by the renter to first find $U_{n}$, for $n \in[1, N]$, taking the computational complexity of 
$\mathcal{O}\left(2(k+1)\left(K_{1}+K_{2}+\cdots+K_{N}\right)\right)$, and then performs tensor modal product along each mode of tensor $\mathcal{X}$, requiring $\mathcal{O}\left((k+1) N\left(K_{1} K_{2} \cdots K_{N}\right)\right)$ computational complexity. In the process of decomposing $\overline{\mathcal{X}}$, the computations are performed by the cloud to decompose $\overline{\mathcal{X}}$, taking $\mathcal{O}\left(N K_{1} K_{2} \cdots K_{N} d+\right.$ $\left.\left(K_{1}+K_{2}+\cdots+K_{N}\right) d^{2}+N d^{3}\right)$ computational complexity at each iteration. In the process of recovering real factor matrices, the computations are conducted by the renter to first obtain $U_{n}^{-1}$, for $n \in[1, N]$, taking the computational complexity of $\mathcal{O}\left((k+1)\left(K_{1}+K_{2}+\cdots+K_{N}\right)\right)$, and then find each real factor matrix, requiring the computations with $\mathcal{O}\left(\left(K_{1}+K_{2}+\cdots+K_{N}\right) d\right)$ complexity.

To sum up, conducting CP tensor decompositions of tensor $\mathcal{X}$ requires the computational complexity of $\mathcal{O}((3(k+1)+$ $\left.N d)\left(K_{1}+K_{2}+\cdots+K_{N}\right)+(k+1) N K_{1} K_{2} \cdots K_{N}\right)$ and $\mathcal{O}\left(N K_{1} K_{2} \cdots K_{N} d+\left(K_{1}+K_{2}+\cdots+K_{N}\right) d^{2}+N d^{3}\right)$ at the renter and cloud sides at each iteration, respectively.

\section{B. Communication Complexity}

For our proposed privacy-preserving outsourcing algorithm, the communication overhead occurs in the following two cases. First, the renter uploads the transformed tensor $\tilde{\mathcal{X}}$ to the cloud, which costs the communication complexity of $\mathcal{O}\left(K_{1} K_{2} \cdots K_{N}\right)$. Second, after conducting CP tensor decompositions on the transformed tensor $\overline{\mathcal{X}}$, the cloud returns the results i.e., $\bar{A}^{(1)}, \bar{A}^{(2)}, \cdots, \bar{A}^{(N)}$ to the renter, which results in the communication complexity of $\mathcal{O}\left(\left(K_{1}+K_{2}+\cdots+K_{N}\right) d\right)$. Thus, our proposed algorithm takes the total communication complexity of $\mathcal{O}\left(K_{1} K_{2} \cdots K_{N}+\left(K_{1}+K_{2}+\cdots+K_{N}\right) d\right)$.

\section{Privacy Analysis}

Inspecting our proposed privacy-preserving outsourcing algorithm, we observe that the cloud can only access to the transformed tensor $\mathcal{X}$, instead of the original one. Hence, we say that the privacy of the data tensor is protected. Moreover, at each iteration, the intermediate results $\bar{A}^{(1)}, \bar{A}^{(2)}, \cdots, \bar{A}^{(N)}$ are obtained by best approximating the transformed tensor. Thus, all these intermediate results cannot reveal any private information of the factor matrices. Finally, the factor matrices the renter obtains are transformed by $A^{(n)} U_{n}$, for $1 \leq n \leq N$. Hence, the privacy of these factor matrices are protected. Therefore, we can say that our proposed privacy-preserving outsourcing algorithm can protect the private information carried by a tensor and its corresponding factor matrices from leaking to third-parties.

\section{EXPERIMENT RESULTS}

To thoroughly evaluate the developed algorithm, we implement our proposed algorithm in a real-world scenario. Specifically, we construct a two-party cloud computing architecture formed by a laptop and EC2 cloud. The laptop has a dualcore $2.7 \mathrm{GHz}$ CPU, 8GB RAM memory, and 256GB solid-state drive and the cloud has 4 computing nodes. We also employ Apache Spark computing framework to manage the storage and computing operations. We implement both the renter-side part and cloud-side part of the proposed algorithm by Python,

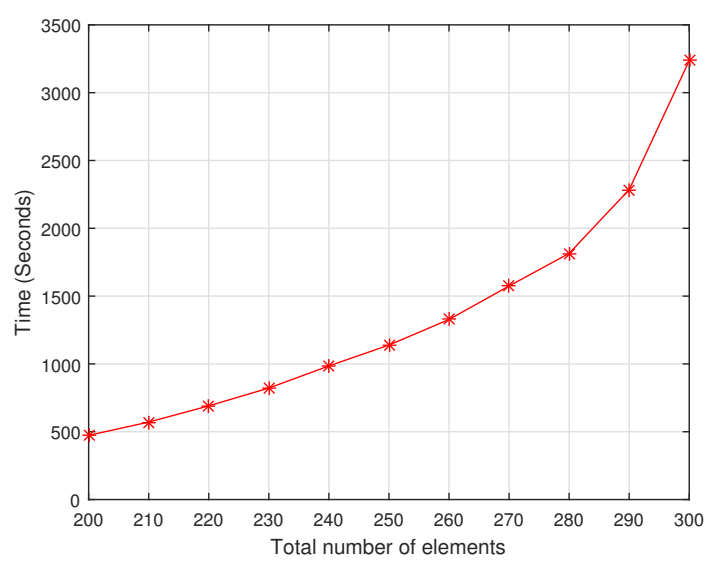

Fig. 2. Computing time of running the renter-side part of the proposed algorithm.

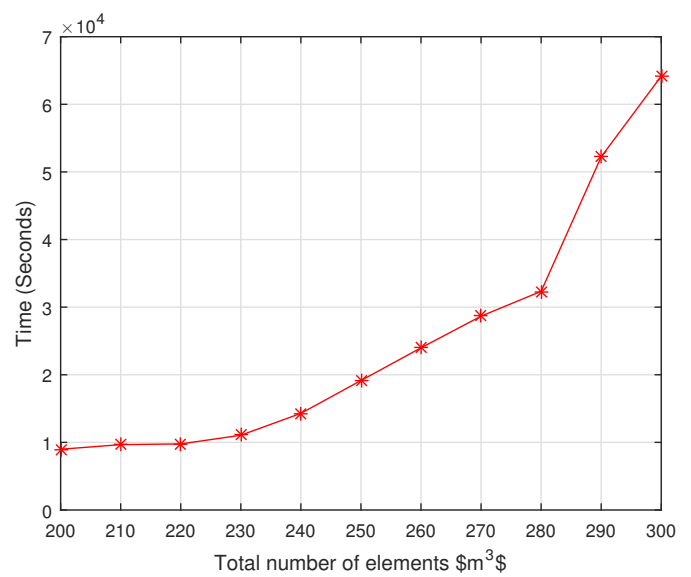

Fig. 3. Computing time of running the cloud-side part of the proposed algorithm.

and let the laptop and EC2 cloud run the renter-side and the cloud-side, respectively. We validate the performance of our proposed algorithm by using real-world data taken from MovieLens Dataset [23]. Besides, we want to decompose a third-order tensor with $K_{1}=K_{2}=K_{3}$.

In this experiment, we measure the computing time of conducting the $\mathrm{CP}$ tensor decompositions of a tensor to assess the performance of our proposed algorithm. Specifically, we compare the computing time spent by running the renter-side part and cloud-side part of the proposed algorithm, and also focus on the computing time spent by the one only locally running the ALS algorithm.

We illustrate by Fig. 2 the measured results at the renter side. The computation time only includes the time it takes to transform the tensor and recover real factor matrices. We can notice that the computation time it spends is very low even for a large-scale tensor. For instance, the time it takes to decompose a $300 \times 300 \times 300$ tensor is $3236.5 s$. In addition, we can find that the measured time is growing up as the tensor size increases.

To compare with the renter, we demonstrate by Fig. 3 the 


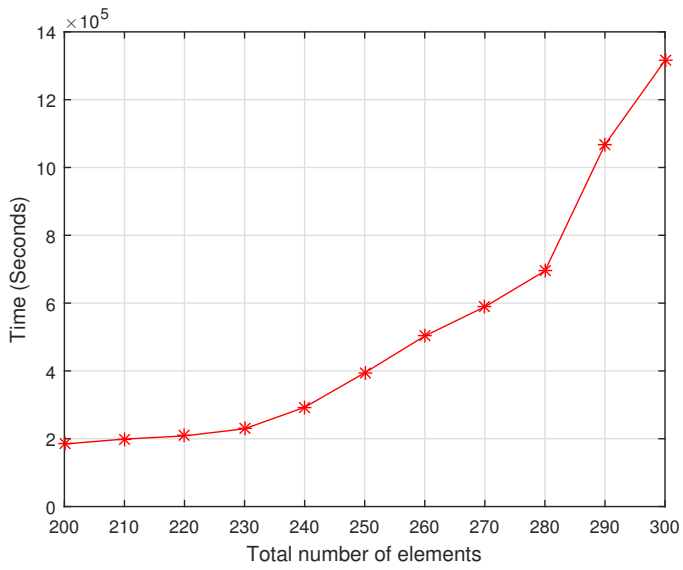

Fig. 4. Computing time of locally performing the $\mathrm{CP}$ decompositions of tensors with different sizes.

measured results we obtained at the cloud side. Specifically, we can observe that the time it takes at the cloud side is increasing with the increasing data size. Moreover, we can find that the spent time still is acceptable, even if the tensor size is very large. For example, decomposing a tensor with $300^{3}$ elements costs $64160.9 \mathrm{~s}$, which is practical for running it in the cloud.

To show the performance improvement by our proposed algorithm, we illustrate by Fig. 4 the measured time the renter spends to solely perform $\mathrm{CP}$ tensor decompositions of tensors with different data sizes. From this figure, we can notice that the measured time is very large. For example, the running time is $1283219 \mathrm{~s}$ when the tensor has $300^{3}$ elements. Besides, the time is growing up as the tensor size increases. This observation urges us to adopt the proposed algorithm to perform large-scale $\mathrm{CP}$ tensor decompositions.

Based on the measured computing time, we can see that our proposed algorithm can save the computing time spent at the renter side very significantly. Moreover, we can find that the proposed algorithm can outsource the most expensive computaitons to the cloud. Therefore, our proposed algorithm is very efficient and practical.

\section{CONCLUSIONS}

In this paper, we have studied the problem of privacypreserving outsourcing of large-scale CP tensor decompositions. To design a privacy-preserving outsourcing algorithm, we develop an efficient privacy-preserving tensor transformation scheme for preserving the private information carried by elements' values of a tensor. Then we design a privacypreserving outsourcing algorithm for the ALS algorithm based $\mathrm{CP}$ tensor decompositions. We also offer a thorough analysis of the performance of our proposed algorithm. To validate the experiment performance, we implement our proposed algorithm on a laptop and the Amazon EC2 cloud. The experiment results have shown that our proposed algorithm is effective in saving the computing time.

\section{REFERENCES}

[1] E. E. Papalexakis, C. Faloutsos, and N. D. Sidiropoulos, "Tensors for data mining and data fusion: Models, applications, and scalable algorithms," ACM Trans. Intelligent Syst. and Tech., vol. 8, pp. 1-44, Dec. 2016.

[2] A. Zanella, N. Bui, A. Castellani, L. Vangelista, and M. Zorzi, "Internet of things for smart cities," IEEE Internet of Things Journal, vol. 1, pp. 22-32, Feb. 2014.

[3] J. C. Ho, J. Ghosh, and J. Sun, "Marble: High-throughput phenotyping from electronic health records via sparse nonnegative tensor factorization," in Proc. ACM SIGKDD'14, (New York, NY, USA), pp. 115-124, Aug. 24 - 272014.

[4] E. Acar, S. A. Camtepe, M. S. Krishnamoorthy, and B. Yener, "Modeling and multiway analysis of chatroom tensors," in Proc. IEEE ISI'05, (Atlanta, GA, USA), pp. 256-268, May 19 - 202005.

[5] A. S. Elmaghraby and M. M. Losavio, "Cyber security challenges in smart cities: Safety, security and privacy," Journal of Advanced Research, vol. 5, pp. 491-497, Jul. 2014.

[6] W. M. Campbell, C. K. Dagli, and C. J. Weinstein, "Social network analysis with content and graphs," Lincoln Laboratory Journal, vol. 20, pp. 62-81, Jan. 2013.

[7] R. A. Harshman, "Foundations of the PARAFAC procedure: Models and conditions for an 'explanatory' multi-modal factor analysis," UCLA Working Papers in Phonetics, vol. 16, pp. 1-84, 1970.

[8] P. Comon, X. Luciani, and A. D. Almeida, "Tensor decompositions , alternating leaster squares and other tales," Journal of Chemometrics, Wiley, vol. 23, pp. 393-405, Aug. 2009.

[9] L. D. Lathauwer and D. Nion, "Decompositions of a higher-order tensor in block terms- part III: alternating least squares algorithms," SIAM Journal on Matrix Anal. and Appl., vol. 30, pp. 1067-1083, Sep. 2008.

[10] L. R. Tucker, "Some mathematical notes on three-mode factor analysis," Psychometrika, vol. 31, pp. 279-311, Sep. 1966.

[11] E. Bertino, B. C. Ooi, Y. Yang, and R. H. Deng, "Privacy and ownership preserving of outsourced medical data," in Proc. IEEE ICDE'05, (Tokyo, Japan), Apr. 5 - 82005.

[12] J. Zhang, F. Wang, K. Wang, W. Lin, X. Xu, and C. Chen, "Data-driven intelligent transportation systems: A survey," IEEE Trans. Intelligent Transportation Systems, vol. 12, pp. 1624-1639, Jul. 2011.

[13] C. Wang, K. Ren, and J. Wang, "Secure and practical outsourcing of linear programming in cloud computing," in Proc. IEEE INFOCOM'11, (Shanghai, China), pp. 880-889, Apr. 10-15 2011.

[14] S. Salinas, C. Luo, X. Chen, and P. Li, "Efficient secure outsourcing of large-scale linear systems of equations," in Proc. IEEE INFOCOM'15, (Hongkong, China), pp. 1035-1043, Apr. 26 - May 12015.

[15] S. Salinas, C. Luo, X. Chen, W. Liao, and P. Li, "Efficient secure outsourcing of large-scale sparse linear systems of equations," IEEE Trans. Big Data, vol. 4, pp. 26-39, Jan.-Mar. 2018.

[16] S. Salinas, C. Luo, W. Liao, and P. Li, "Efficient secure outsourcing of large-scale quadratic programs," in Proc. ACM Asia CCS'16, (Xi'an, Shaanxi, China), 30 May - 1. Jun 2016.

[17] C. Luo, K. Zhang, S. Salinas, and P. Li, "Secfact: Secure large-scale QR and LU factorizations," IEEE Trans. Big Data, vol. PP, pp. 1-13, Dec. 2017.

[18] C. Luo, K. Zhang, S. Salinas, and P. Li, "Efficient privacy-preserving outsourcing of large-scale QR factorization," in Proc. IEEE BigDataSE, (Sydney, Australia), pp. 917-924, Aug. 2017.

[19] C. Luo, J. Ji, X. Chen, M. Li, L. T. Yang, and P. Li, "Parallel secure outsourcing of large-scale nonlinearly constrained nonlinear programming problems," IEEE Trans. Big Data, vol. PP, pp. 1-13, Mar. 2018.

[20] L. Zhou and C. Li, "Outsourcing eigen-decomposition and singular value decomposition of large matrix to a public cloud," IEEE Access, vol. 4, pp. 869-879, Apr. 2017.

[21] W. Liao, W. Du, S. Salinas, and P. Li, "Efficient privacy-preserving outsourcing of large-scale convex separable programming for smart cities," in IEEE 14th International Conference on Smart City, pp. 13491356, IEEE, 2016.

[22] W. Liao, C. Luo, S. Salinas, and P. Li, "Efficient secure outsourcing of large-scale convex separable programming for big data," IEEE Transactions on Big Data, 2017.

[23] F. M. Harper and J. A. Konstan, "The MovieLens Datasets: History and Context," ACM Trans. Interactive Intelligent Syst., vol. 5, pp. 1-19, Dec. 2015. 